\title{
A Case of Thymoma with Paraneoplastic Neurologic Disorders
}

\author{
Authors \\ Sudhir Kumar Atri ${ }^{1}$, Mohini ${ }^{1}$, Neeraj Kumar ${ }^{1}$, Gaurav Rathee ${ }^{1 *}$, Vinay Singla ${ }^{1}$, \\ Homdutt Sharma ${ }^{1}$, Devender Yadav ${ }^{1}$ \\ ${ }^{1}$ Department of Medicine, Pt. B.D. Sharma PGIMS, Rohtak, Haryana - 124001, India \\ *Corresponding Author \\ Gaurav Rathee \\ Resident, Department of Medicine, Pt. B.D. Sharma PGIMS, Rohtak, Haryana - 124001, India \\ Email: gauravrathee@hotmail.com
}

\begin{abstract}
Thymomas are well known for paraneoplastic neurologic disorders (PNDs), but multiple PNDs co-exist rarely. We are reporting a case of thymoma with myasthenia gravis who presented with concomitant status epilepticus, likely of paraneoplastic etiology, without any evidence of encephalitis, on imaging, and on cerebrospinal fluid (CSF) examination. Possibly, a case of two paraneoplastic autoimmune disorders coexisting in a thymoma patient.

Keywords: Thymoma, Myasthenia Gravis, Seizures, Paraneoplastic Neurologic Disorders.
\end{abstract}

\section{Introduction}

Paraneoplastic neurologic disorders (PNDs) are a group of syndromes, related to neoplasia, that affect the nervous system, mostly through immune mechanisms. PNDs occur in $0.5-1 \%$ of all cancer patients, whereas in thymoma patients, occurrence of PNDs is very high, to the tune of $30-50 \%{ }^{1}$. The thymus is an important organ for development of immune tolerance. T-cells with strong affinity to self-antigens are eliminated, and thus selection of T-cells with appropriate self-tolerance occurs in thymus. Hence, it is not surprising to see PNDs in thymoma patients. PNDs well known to be associated with thymoma include Myasthenia gravis (MG), Lambert Eaton Myasthenic syndrome (LEMS), acquired Neuromyotonia (aNMT), Morvan's syndrome, Encephalitis,
Seizures and Myositis. Most of these disorders have an established autoimmune basis, and autoantibody markers have been detected. Patients manifesting with such disorders should be screened for thymoma at diagnosis. These disorders can cause profound disability but usually respond to immunotherapy, and often improve with thymectemy. Myasthenia gravis (MG) is by far the commonest among these PNDs. About 15 to $20 \%$ of MG patients have a thymoma. On the other hand, $24.5-40 \%$ of thymoma patients develop $\mathrm{MG}^{2-5}$. Rarely, multiple, co-existing autoimmune syndromes are known to occur in thymoma patients. About $15-20 \%$ of patients with Caspr2 Ab (contactin-associated protein-like 2 antibody) syndromes have MG that may occur before, after, or during the presentation of their 
encephalitis or aNMT $^{6}$. Such diverse paraneoplastic syndromes are difficult to diagnose. These patients may have different autoimmune disorders at the same time, or in chronological order, separated by months. When two autoimmune syndromes occur simultaneously, they may present a complex picture, very difficult to diagnose. For example, a patient with bulbar weakness from $\mathrm{MG}$, and hyper-reflexia, and diffuse fasciculations from aNMT may give an impression of motor neuron disease $^{6-8}$.

Immune-mediated encephalitides are also being recognized as casue of seizures and status epilepticus, which may have a paraneoplastic etiology. In past, cases have been reported, where thymomas have been associated with limbic or cortical encephalitis. Recently, a few cases of thymoma associated pan-encephalitis have also been reported ${ }^{9}$. In majority of the reported patients of thymoma associated encephalitides, neurologic manifestations included memory loss, confusion, and seizures. $70 \%$ of reported patients had bilateral non-enhancing hyperintensity on brain MRI, some appearing weeks after the onset of symptoms. Finding a thymoma in these patients would suggest a PND ${ }^{10}$. Detection of neuronal or neuromuscular junction autoantibodies, helps in confirming the diagnosis, but they are found in only $60-70 \%$ of PNDs of the $\mathrm{CNS}^{1}$.

\section{Case Description}

A 35 years old female presented in Emergency Room, with complaints of drooping of both eyelids, intermittent double vision which started about one week ago, and over 2-3 days progressed on to develop dysphagia, nasal regurgitation, slurring of speech with nasal twang, decreased tone of voice, weakness all four limbs, followed by development of dyspnea since last 2-3 days. All the symptoms have been progressively worsening and patient was having dyspnea at rest at the time of presentation. Symptoms used to be relieved partially by rest but recurs on minimal exertion. There was h/o similar illness 4 months ago, but symptoms were mild, and patient had consulted a local practitioner, patient recovered over a week, and was not on any medications. There were h/o episodes of mild shortness of breath for 4-5 years, which used to occur occasionally, for which patient used to took emergency treatment in form of injections, from local practitioner, and symptoms used to be relieved after that treatment. Patient believed that it was due to the dust allergy.

On presentation, the patient was conscious and oriented to time, place, and person. $\mathrm{BP}=110 / 70$ $\mathrm{mmHg}, \mathrm{HR}=96 \mathrm{bpm}, \mathrm{RR}=30 \mathrm{bpm}$, afebrile. Higher mental functions were intact; Sensory examination was within normal limits; Ptosis, Extraocular muscle weakness, facial and pharyngeal weakness were present. Power was $4 / 5$ in all four limbs. Deep Tendon reflexes were within normal limit. On investigation, Acetyl choline Receptor (AChR) antibodies were present. CT thorax (Fig.1) was suggestive of thymoma. She was taken on the ventilator support. Plasmapheresis was done. While recovering she developed super refractory status epilepticus. Patient was started on multiple anti-epileptic drugs with inadequate control. MRI brain was within normal limits. CSF examination was within normal limit. Antibody screening for autoimmune encephalitis (Table-1) was done in CSF, and came out to be negative for (1) anti-AMPAR1, (2) antiAMPAR2, (3) anti-GABA $A_{B}$, (4) anti-LGI-1 (VGKC type), (5) anti-CASPR2 (VGKC type), (6) anti-NMDAR. Patient has improved in terms of weakness, and in terms of seizures, but seizures are still not well controlled yet, and she has become bedridden. Now, she is on low dose steroids, and thymectomy has been advised. 


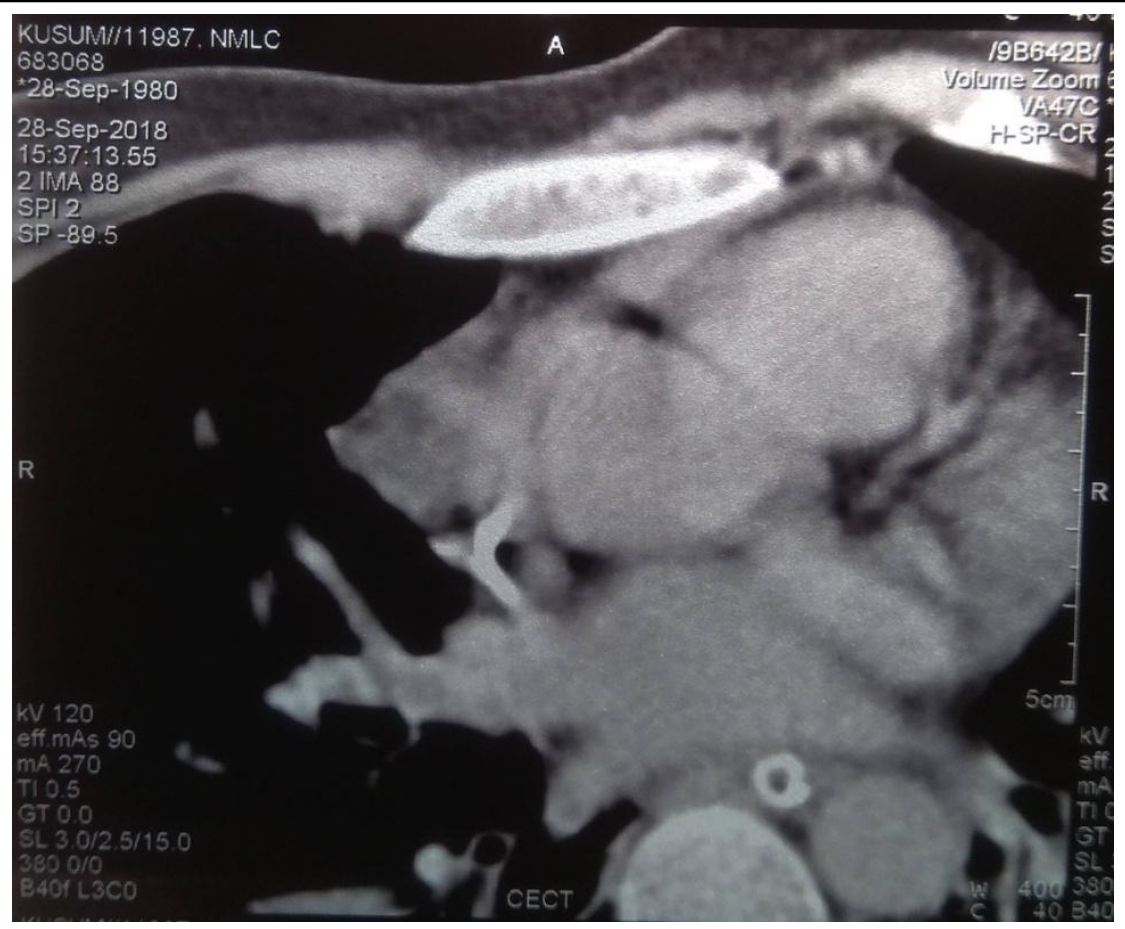

Fig.1 Axial section of Computed Tomography thorax showing anterior mediatinal mass

Table 1 Antibodies tested in this patient to screen for paraneoplastic disorders

\begin{tabular}{|c|c|}
\hline Antibody & \multicolumn{1}{|c|}{ Result } \\
\hline Anti-AChR (muscle) & Positive \\
\hline Anti-AMPAR1 & Negative \\
\hline Anti-AMPAR2 & Negative \\
\hline Anti-GABA ${ }_{\mathrm{B}} \mathrm{R}$ & Negative \\
\hline Anti-LGI1 (VGKC type) & Negative \\
\hline \multicolumn{1}{|c|}{ Negative } \\
\hline Anti-CASPR2 antibody (VGKC type) & Negative \\
\hline $\begin{array}{l}\text { Abbreviations: AChR, acetylcholine receptor; AMPA, alpha-amino-3-hydroxy-5-methyl-4- } \\
\text { isoxazolepropionic acid receptor; GABA }{ }_{\mathrm{B}} \mathrm{R}, \text { gamma-amino butyric acid B receptor; LGI, } \\
\text { Leucine-rich glioma-inactivated 1; CASPR2, cantactin-associated protein-like 2; VGKC, } \\
\text { Voltage gated potassium channel; NMDAR, N-methyl-D-aspartate receptor. }\end{array}$ \\
\hline
\end{tabular}

Table 2 Guidelines for the diagnosis of Paraneoplastic Neurologic Disorders ${ }^{2}$

\begin{tabular}{|l|}
\hline Guidelines for the Diagnosis of Paraneoplastic Neurologic Disorders \\
\hline Definite Paraneoplastic Neurologic Disorder \\
\hline 1. A classic syndrome and cancer \\
2. A nonclassic syndrome that resolves or significantly improves after cancer treatment \\
3. A nonclassic syndrome with paraneoplastic antibodies (well characterized or not) and \\
cancer \\
4. A neurologic syndrome (classic or not) with well-characterized antibodies and no \\
detected cancer \\
1. A classic syndrome, no paraneoplastic antibodies, and no cancer, but at high risk to have \\
an underlying tumor \\
A neurologic syndrome (classic or not) with partially characterized paraneoplastic \\
3. A nonclassic syndrome with cancer but without paraneoplastic antibodies \\
Modified from Graus F, Delattre JY, Antoine JC, et al. Recommended diagnostic criteria for \\
paraneoplastic neurological syndromes. J Neurol Neurosurg Psychiatry 2004;75(8):1135Y1140. B \\
2004, with permission from BMJ Publishing Group Ltd. jnnp.bmj.com/content/75/8/1135.full. \\
\hline
\end{tabular}




\section{Discussion}

This patient presented with myasthenic crisis, CT imaging revealed the thymoma, and within onemonth patient also developed super refractory status epilepticus. Over a short period of time, patient had become bedridden. There was no evidence of encephalitis, on imaging and CSF examination. The Myasthenia Gravis and the super refractory seizures both appeared to be paraneoplastic phenomena related to the thymoma, casused through autoimmune mechanisms. This patient was positive for Acetyl Choline Receptor antibodies, which confirmed the diagnosis of myasthenia gravis In view of the possible autoimmune etiology of seizures, screening was done for several antibodies known to cause seizures (Table 1). Patient came out to be negative for anti-AMPAR1, anti-AMPAR2, anti$\mathrm{GABA}_{\mathrm{B}} \mathrm{R}$, anti-LGI-1(VGKC type), anti-CASPR2 (VGKC type), anti-NMDAR. Guidelines for the diagnosis of PNDs have been developed on the basis of the type of neurologic syndrome, detection of cancer, and presence or absence of paraneoplastic antibodies, with the caveat that the tumor diagnosis and the development of the neurologic syndrome should occur within 5 years of each other (Table 2) , $^{21}$. According to these guidelines, this case fits into the diagnosis of Definite Paraneoplastic Neurological Disorder for Myasthenia Gravis. In addition, the super refractory seizures also seem to be of paraneoplastic etiology, fitting into the category of Possible Paraneoplastic Neurological Disorder, as we have not been able to find any associated antibody yet.

It has been shown that immunological responses to synaptic receptors or associated cell surface proteins can cause seizures, which are often refractory to antiepileptic drugs, unless the immune mechanism is identified and treated. Status epilepticus may be the first manifestation of such a disease, and the patient might need deep sedation, leading to suppression of other symptoms and thus causing difficulty and delay in recognizing the syndromes. The identification of many such potentially treatable autoimmune disorders, has led to increased recognition of cases with encephalitis and refractory seizures, in which the antigens are unknown. In this patient, we need to do further tests for other autoantibodies, known for causing seizures, including anti-GABA $A_{A} R$ (gamma-amino butyric acid $\mathrm{A}$ receptor) antiGAD-65 (Glutamic acid decarboxylase-65), antiCRMP5 (Collapsin response-mediator protein 5). A recent study has shown that $\mathrm{GABA}_{\mathrm{A}} \mathrm{R}$ antibodies are associated with a severe form of encephalitis with seizures, refractory status epilepticus or both. These antibodies have also been shown to have downregulating effect on receptors in cultured neurons ${ }^{12,13}$. In addition, further research may uncover additional novel antibodies for such seronegative cases and thus, improve our ability to diagnose these patients. Till now this patient has not undergone thymectomy, and thus the effects of removing the thymectomy on her symptoms are not yet known. Review of literature suggests that, almost all such PND patients, who had received thymectomy, have shown good response (partial or full), in terms of improvement in MG symptoms, as well as, resolution of status epilepticus and decrease in antibody titers. Even in non-thymoma MG patients, thymectomy has also been advocated as a treatment option. A recent randomized clinical trial has shown that thymectomy improves the clinical outcomes, and decrease the need of immunosuppressive therapy in myasthenia gravis patients $^{14}$.

\section{Conclusion}

Paraneoplastic etiology should be suspected in patients with diseases like MG, and work up should be done to find the related neoplasia. Similarly, in a patient presenting with seizures, possibility of autoimmune encephalitis should be considered, and screening for autoantibodies may be done, if there are other features suggesting possibility of autoimmune etiology. Rarely, multiple PNDs may co-exist in a thymoma patient. If thymoma is found as a cause of the 
PNDs, early surgical removal of the tumor should be advised, as it would provide double benefit, in terms of tumor treatment, and as well as, patient will possibly get relieved of the paraneoplastic symptoms.

\section{References}

1. Dalmau J, Rosenfeld M R. Paraneoplastic Neurologic Syndromes and Autoimmune Encephalitis. Chapter 90. Harrison's Principles of Internal Medicine. 20th Edition. McGraw-Hill Education,

2. Rosenfeld M R, Dalmau J O. Paraneoplastic disorders of the CNS and autoimmune synaptic encephalitis. Continuum Lifelong Learning Neurol 2012; 18(2): 366-383

3. Marx A, Pfister F, Schalke B, SaruhanDireskeneli G, Melms A, Strobel P. The different roles of the thymus in the pathogenesis of the various myasthenia gravis subtypes. Autoimmun Rev. 2013; 12(9): 875-884.

4. Dzhagalov I, Phee H. How to find your way through the thymus: a practical guide for aspiring T cells. Cell Mol Life Sci. 2012; 69(5): 663-682.

5. Gadalla S M, Rajan A, Pfeiffer R, et al. A population-based assessment of mortality and morbidity patterns among patients with thymoma. Int J Cancer. 2011; 128(11): 2688-2694.

6. Lancaster E, Huijbers MG, Bar V, et al. Investigations of caspr2, an autoantigen of encephalitis and neuromyotonia. Ann Neurol. 2011; 69(2): 303-311.

7. Diaz-Manera J, Rojas-Garcia R, Gallardo E, et al. Antibodies to AChR, MuSK and VGKC in a patient with myasthenia gravis and Morvan's syndrome. Nat Clin Pract Neurol. Jul; 2007; 3(7): 405-410

8. Geddes, J. A Case of Invasive Thymoma. The Medicine Forum. 2003; 4: Article 10.
9. Nwabuobi LA, Pellinen JC, Wisniewski TM. Thymoma-associated panencephalitis: a newly emerging paraneoplasticneurologic syndrome.

Neuroimmunol Neuroinflammation 2017; 4: 117-23.

10. Erkmen C P, Fadul C E, Dalmau J, Erkmen K. Thymoma-associated paraneoplastic encephalitis (TAPE): Diagnosis and treatment of a potentially fatal condition. J Thorac Cardiovasc Surg 2011;141: e17-20

11. Graus F, Delattre J Y, Antoine J C, Dalmau J, Giometto B, Grisold W, Honnorat J, Sillevis Smitt P, Vedeler Ch, Verschuuren J J G M, Vincent A, Voltz R, for the Paraneoplastic Neurological Syndrome Euronetwork. Recommended diagnostic criteria for paraneoplastic neurological syndromes. J Neurol Neurosurg Psychiatry 2004; 75: 1135-1140.

12. Petit-Pedrol $M$, Armangue $T$, Peng $X$, Bataller L, Cellucci T, Davis R, McCracken L, Martinez-Hernandez E, Mason WP, Kruer MC, RitaccoDG, Grisold W, Meaney BF, Alcala C, Sillevis-Smitt P, Titulaer M J, Balice-Gordon R, Graus F, Dalmau J. Encephalitis with refractory seizures, status epilepticus, and antibodies to the GABAA receptor: a case series, characterisation of the antigen, and analysis of the effects of the antibodies. Lancet Neurol 2014;13: 276-86.

13. Okhawa T, Satake S, Yokoi N, Miyazaki Y, Ohshita T, Sobue G, Takashima H, Watanabe O, Fukata Y, Fukata M. Identification and characterization of GABAa receptor autoantibodies in autoimmune encephalitis. J Neurosci 2014; 34: 8151-63.

14. Wolfe G I, Kaminski H J, Minisman G, et al, for the Myasthenia Gravis Patients Receiving Prednisone Therapy (MGTX) Study Group. Randomized Trial of Thymectomy in Myasthenia Gravsis. N Engl J Med 2016; 375:511-522. 